hep-th/0606048

\title{
D-Brane Amplitudes in Topological String on Conifold
}

\author{
Kazumi Okuyama \\ Department of Physics and Astronomy, University of British Columbia \\ Vancouver, BC, V6T 1Z1, Canada \\ kazumi@phas.ubc.ca
}

\begin{abstract}
We study the relation between two kinds of topological amplitudes of non-compact Dbranes on conifold. In the A-model, D-branes are represented by fermion operators in the melting crystal picture and the amplitudes are given by the quantum dilogarithm. In the mirror B-model, D-branes correspond to the determinant operator $\operatorname{det}(x-M)$ in the ChernSimons matrix model and the amplitudes are given by the Stieltjes-Wigert polynomial. We show that these two amplitudes are related by a certain integral transformation. We argue that this transformation represents the deformation of closed string background due to the presence of D-branes.
\end{abstract}

June 2006 


\section{Introduction}

The gauge/string duality is one of the most important aspects of string theory. It appears that this duality is also essential for the topological string theory. In [1],2], it is realized that the 't Hooft expansion of Chern-Simons theory on $S^{3}$ is exactly the same as the topological A-model on the resolved conifold $\mathcal{O}(-1) \oplus \mathcal{O}(-1) \rightarrow \mathbb{P}^{1}$. It was shown that the partition function of $S U(N)$ level $k$ Chern-Simons theory on $S^{3}$ [3]

$$
Z_{C S}=\frac{1}{(k+N)^{\frac{N}{2}}} \prod_{\alpha>0} 2 \sin \frac{\pi(\alpha, \rho)}{k+N}
$$

agrees with the partition function of topological closed string on local $\mathbb{P}^{1}$ [4]

$$
Z_{\text {top }}=\prod_{n=1}^{\infty}\left(\frac{1-Q q^{n}}{1-q^{n}}\right)^{n}
$$

up to a non-perturbative factor $\eta(q)^{N}$. In this correspondence, various parameters are related as

$$
\begin{gathered}
g_{s}=\frac{2 \pi i}{k+N}, \quad t=g_{s} N, \\
q=e^{-g_{s}}, \quad Q=e^{-t},
\end{gathered}
$$

where $g_{s}$ is the string coupling and the 't Hooft parameter $t$ is identified as the Kähler parameter of $\mathbb{P}^{1}$. This is an example of geometric transition where D-branes wrapped around $S^{3}$ in the deformed conifold [5] is replaced by a $B$-field on $S^{2}$ in the resolved conifold. In [6], this duality was derived by using a linear sigma model.

In this paper, we consider open string amplitudes associated with non-compact Dbranes in this background. There are two ways to define this amplitude. The first way is to introduce non-compact Lagrangian A-branes in the deformed conifold $T^{*} S^{3}$. The amplitude of the A-branes is given by Wilson loops in Chern-Simons theory [7]. Later it was shown that the same amplitude is obtained in the melting crystal picture 8,9]. In this picture, the A-brane corresponds to a defect of crystal and it is represented by a certain fermion operator $\Psi_{D}(x)$ [10]. The amplitude $\left\langle\Psi_{D}(x)\right\rangle$ is given by the quantum dilogarithm 10 [13].

The second way to define the D-brane amplitude is to use the matrix model description of Chern-Simons theory [14 17]. A natural D-brane amplitude in the Chern-Simons matrix model is the expectation value of determinant $\langle\operatorname{det}(x-M)\rangle$. Since this matrix model appears as the mirror B-model of conifold [15], the determinant $\operatorname{det}(x-M)$ represents a 
non-compact B-brane in the mirror description [18]. It is well known that $\langle\operatorname{det}(x-M)\rangle$ is equal to the $N$-th orthogonal polynomial $P_{N}(x)$. For the Chern-Simons matrix model, the associated orthogonal polynomial is known as the Stieltjes-Wigert polynomial [19].

It is natural to ask what is the relation between the A-brane amplitude $\left\langle\Psi_{D}(x)\right\rangle$ in the crystal picture and the B-brane amplitude $\langle\operatorname{det}(x-M)\rangle$ in the Chern-Simons matrix model. In this paper, we will show that they are related by a simple Gaussian integral.

This paper is organized as follows. In section 2, we review the Gaussian matrix model as a preliminary of the analysis of Chern-Simons matrix model. In section 3, we find a simple relation between A-brane amplitudes and B-brane amplitudes. Section 4 is discussion.

\section{Gaussian Matrix Model: Review}

Before discussing the D-branes in topological string, let us first review a simple example, namely FZZT-branes in Gaussian matrix model [20]. Although this is a too trivial example, we will see that most of the properties found in the Gaussian matrix model have close analogues in the Chern-Simons matrix model.

The Gaussian matrix model is defined by

$$
Z_{G}=\int_{N \times N} d M e^{-\frac{1}{2 g_{s}} \operatorname{Tr} M^{2}}
$$

We are interested in the expectation value of the determinant operator $\operatorname{det}(x-M)$

$$
\langle\operatorname{det}(x-M)\rangle=\frac{1}{Z_{G}} \int d M e^{-\frac{1}{2 g_{s}} \operatorname{Tr} M^{2}} \operatorname{det}(x-M),
$$

which can be interpreted as the wavefunction of FZZT-brane. As discussed in detail in [20], this integral (2.2) is evaluated by rewriting the determinant as a fermion integral and then integrate out $M$. The resulting expression is an effective theory on the single FZZT-brane

$$
\langle\operatorname{det}(x-M)\rangle=\int_{-\infty}^{\infty} \frac{d s}{\sqrt{2 \pi g_{s}}} e^{-\frac{1}{2 g_{s}} s^{2}} f_{N}(x+i s) .
$$

Here we introduced the function $f_{N}(x)=x^{N}$. We can easily see that (2.3) is nothing but the integral representation of the Hermite polynomial, which in turn is the $N$-th orthogonal polynomial $P_{N}(x)$ of Gaussian measure

$$
\langle\operatorname{det}(x-M)\rangle=P_{N}(x)=\left(\frac{g_{s}}{2}\right)^{\frac{N}{2}} H_{N}\left(\frac{x}{\sqrt{2 g_{s}}}\right) .
$$


It is interesting that $f_{N}(x)=x^{N}$ can be thought of as a "classical" value of the determinant. Namely, $f_{N}(x)$ is the value of $\operatorname{det}(x-M)$ evaluated at the minimum of the Gaussian potential $\operatorname{Tr} M^{2}$

$$
f_{N}(x)=\operatorname{det}\left(x-M_{0}\right), \quad M_{0}=\operatorname{diag}(0,0, \cdots, 0) .
$$

From this viewpoint, (2.3) defines an integral transformation $\mathcal{Q}$ which maps the "classical wavefunction" $f_{N}(x)$ into the "quantum wavefunction" $P_{N}(x)$

$$
\begin{aligned}
& \mathcal{Q}: \operatorname{det}\left(x-M_{0}\right) \mapsto\langle\operatorname{det}(x-M)\rangle \\
& (\mathcal{Q} f)(x)=\int_{-\infty}^{\infty} \frac{d s}{\sqrt{2 \pi g_{s}}} e^{-\frac{1}{2 g_{s}} s^{2}} f(x+i s)
\end{aligned}
$$

Note that this relation holds for arbitrary $N$, therefore $f_{n}(x)=x^{n}$ is mapped to $P_{n}(x)$ for all $n$

$$
\mathcal{Q}: f_{n}(x) \mapsto P_{n}(x)
$$

We can generalize this picture to the multi-point correlators of FZZT-branes. Following the similar procedure as above, the $K$-point function of determinants is written as a $K \times K$ matrix model [20]

$$
\left\langle\prod_{i=1}^{K} \operatorname{det}\left(x_{i}-M\right)\right\rangle=\int_{K \times K} d S e^{-\frac{1}{2 g_{S}} \operatorname{Tr} S^{2}} \operatorname{det}(X+i S)^{N}
$$

where $X=\operatorname{diag}\left(x_{1}, \cdots, x_{K}\right)$. This can be thought of as an effective open string theory on the $K$ FZZT-branes. After integrating out the angular part of $S$, (2.8) becomes an integral over the $K$ eigenvalues of $S$

$$
\Delta(x)\left\langle\prod_{i=1}^{K} \operatorname{det}\left(x_{i}-M\right)\right\rangle=\int \prod_{k=1}^{K} \frac{d s_{k}}{\sqrt{2 \pi g_{s}}} e^{-\frac{1}{2 g_{s}} s_{k}^{2}} f^{(K)}\left(x_{1}+i s_{1}, \cdots, x_{K}+i s_{K}\right) .
$$

Here $\Delta(x)=\prod_{i>j}\left(x_{i}-x_{j}\right)$ is the Vandermonde determinant and $f^{(K)}(x)$ is given by

$$
f^{(K)}\left(x_{1}, \cdots, x_{K}\right)=\Delta(x) \prod_{i=1}^{K} f_{N}\left(x_{i}\right) .
$$

1 After taking a double scaling limit, this open string theory on the FZZT-branes is identified as the Kontsevich model [21,20]. 
As before, we regard $f^{(K)}(x)$ as a "classical" value of the $K$-point function. The true correlator of determinants is given by the $\mathcal{Q}$-transform of $f^{(K)}(x)(2.9)$.

To evaluate the integral (2.9), we rewrite $f^{(K)}(x)(2.10)$ as

$$
f^{(K)}(x)=\operatorname{det}\left(x_{i}^{j-1}\right) \prod_{i=1}^{K} x_{i}^{N}=\operatorname{det}\left(x_{i}^{N+j-1}\right)=\operatorname{det}\left(f_{N+j-1}\left(x_{i}\right)\right) .
$$

Now the integral (2.9) is easily evaluated by recalling that the integral transformation $\mathcal{Q}$ maps $f_{n}(x)$ into $P_{n}(x)(2.7)$. Therefore, the $K$-point correlator of FZZT-brane is given by

$$
\Delta(x)\left\langle\prod_{i=1}^{K} \operatorname{det}\left(x_{i}-M\right)\right\rangle=\operatorname{det}\left(P_{N+j-1}\left(x_{i}\right)\right) .
$$

As expected, this agrees with the general formula of the correlator of determinants [22]. In (2.12) we have multiplied the Vandermonde determinant $\Delta(x)$ to the correlator of determinants. This factor makes the left hand side of (2.12) anti-symmetric in $x_{i}$, therefore FZZT-branes become fermionic [20]. To summarize, we found the following relation

$$
\mathcal{Q}: \quad \Delta(x) \prod_{i=1}^{K} \operatorname{det}\left(x_{i}-M_{0}\right) \mapsto \Delta(x)\left\langle\prod_{i=1}^{K} \operatorname{det}\left(x_{i}-M\right)\right\rangle .
$$

In the next section, we will see that the transformation $\mathcal{Q}$ is exactly what we are looking for, i.e., $\mathcal{Q}$ maps A-brane amplitudes into B-brane amplitudes.

\section{D-brane Amplitudes on Conifold}

\subsection{A-branes in the Calabi-Yau Crystal Picture}

As shown in [8,9], the target space theory of topological A-model is reformulated as a certain $U(1)$ gauge theory and it leads to the quantum foam picture of Kähler gravity, which in turn is related to the statistical model of crystal melting. In the case of resolved conifold, the relevant crystal is identified in [11]. It is given by a lattice in positive octant of $\mathbb{R}^{3}$ with lattice spacing $g_{s}$, and it is restricted to the interval $\left[0, N g_{s}\right]$ in one direction. This statistical problem is solved by slicing the $3 \mathrm{D}$ partition into a sequence of $2 \mathrm{D}$ partitions and use the relation between 2D Young diagram and free fermion [8].

In this crystal picture, a non-compact A-brane is represented by a half-line in $\mathbb{R}^{3}$ and it creates a defect in the lattice [10]. It is further shown that the presence of defect is represented by the insertion of a certain fermion operator $\Psi_{D}$ in the free fermion computation 
mentioned above. If the half line associated with the $i$-th D-brane ends on one of the axis of $\mathbb{R}_{+}^{3}$ at $a_{i}=g_{s}\left(N_{i}+\frac{1}{2}\right)$, we identify the moduli $x_{i}$ of this D-brane as 2

$$
x_{i}=q^{N_{i}} .
$$

We are interested in the amplitude of Lagrangian A-brane with moduli $x$

$$
\mathcal{Z}_{N}(x)=\left\langle\Psi_{D}(x)\right\rangle .
$$

This is first obtained by a Wilson loop calculation in Chern-Simons theory on $S^{3}$ [7], and later it is reproduced by the melting crystal picture 10,11,13. We will consider an A-brane with the corresponding half-line ending on the interval $\left[0, N g_{s}\right]$. Then the A-brane has a topology $S^{1} \times \mathbb{R}^{2}$ [23,24, 10]. The explicit form of the single brane amplitude is given by

$$
\mathcal{Z}_{N}(x)=\prod_{n=1}^{N}\left(1-x q^{n}\right)=\frac{L(x, q)}{L(x Q, q)}
$$

where $L(x, q)$ is the quantum dilogarithm [25]

$$
L(x, q)=\prod_{n=1}^{\infty}\left(1-x q^{n}\right)
$$

Similarly, the amplitude in the presence of $K$ A-branes is given by 10,11, 13

$$
Z\left(x_{1}, \cdots, x_{K}\right)=\prod_{i<j}\left(1-x_{i} x_{j}^{-1}\right) \prod_{i=1}^{K} \mathcal{Z}_{N}\left(x_{i}\right)=\Delta(x) \prod_{i=1}^{K} \mathcal{Z}_{N}\left(x_{i}\right) \prod_{i=1}^{K} x_{i}^{1-i}
$$

Although it is a little bit ad hoc, it seems natural to drop the last factor $\prod_{i} x_{i}^{1-i}$ in order to make the multi-point function of D-branes anti-symmetric under the interchange of $x_{i}$ ' 33 . This is motivated by the intuition that non-compact D-branes are fermions. Then the $K$-point function of D-brane operators becomes

$$
\left\langle\prod_{i=1}^{K} \Psi_{D}\left(x_{i}\right)\right\rangle=\Delta(x) \prod_{i=1}^{K} \mathcal{Z}_{N}\left(x_{i}\right)
$$

One can immediately notice the similarity of this expression to the "classical" correlation function (2.10) in the Gaussian matrix model. We would like to argue that this is not just a coincidence. We will see below that the "quantum" version of (3.6) is given by the correlator of determinants in the Chern-Simons matrix model.

2 This definition of $x_{i}$ defers from the usual convention by a factor of $q^{\frac{1}{2}}$. Our definition is convenient when comparing to the B-brane amplitude in the next section.

3 Perhaps this factor might be canceled by carefully analyzing the zero-mode of boson appearing in the bosonization of fermion. 


\subsection{B-branes in Chern-Simons Matrix Model}

In this subsection, we will consider the D-brane amplitudes in the Chern-Simons matrix model. In [14], it is observed that the partition function of Chern-Simons theory is written as a matrix integral. It is then realized that this Chern-Simons matrix model naturally appears in the B-model mirror of resolved conifold [15].

Let us recall the derivation of Chern-Simons matrix model. Using the Weyl denominator formula, it is easy to see that the partition function of Chern-Simons theory (1.1) is written as an integral

$$
Z_{C S}=\int \prod_{i=1}^{N} d u_{i} \prod_{i<j}\left(2 \sinh \frac{u_{i}-u_{j}}{2}\right)^{2} e^{-\frac{1}{2 g_{s}} \sum_{i} u_{i}^{2}}
$$

This is almost a matrix model integral, but the measure factor is not the usual Vandermonde determinant. By the following change of variables [26,16]

$$
m_{i}=e^{u_{i}} q^{-N}
$$

we can change the measure factor in (3.7) into the usual Vandermonde determinant $\Delta(m)^{2}$. Then $Z_{C S}$ becomes an $N \times N$ hermitian matrix model with log-squared potential

$$
Z_{C S}=\int d M e^{-\frac{1}{2 g_{s}} \operatorname{Tr}(\log M)^{2}}
$$

Now let us consider the D-branes in this model. From the general argument [18], non-compact B-branes correspond to the insertion of determinant operators in the matrix model. We define the B-brane operator with moduli $x$ as

$$
B(x)=(-1)^{N} q^{N^{2}+\frac{1}{2} N} \operatorname{det}(x-M)=\operatorname{det}\left(q^{N+\frac{1}{2}} M-q^{N+\frac{1}{2}} x\right) .
$$

We have put a $q$-dependent prefactor for later convenience. This is partly motivated by the relation (3.8) that $\log \left(q^{N} m_{i}\right)$ is the natural Gaussian variable $u_{i}$ in the original integral (3.7). The additional factor $q^{\frac{1}{2}}$ is related to our definition of D-brane moduli $x$ (see footnote 2). Then the B-brane amplitude is given by

$$
\mathcal{S}_{N}(x)=\langle B(x)\rangle
$$

where the expectation value is taken in the Chern-Simons matrix model (3.9). From the Heine's formula, the expectation value of determinant is the $N$-th orthogonal polynomial. 
In the case of log-normal measure (3.9), the orthogonal polynomial is known as the StieltjesWigert polynomial [19] .4 With our normalization of $B(x)$ (3.10), the $N$-th Stieltjes-Wigert polynomial is given by

$$
\mathcal{S}_{N}(x)=\sum_{k=0}^{N}\left[\begin{array}{c}
N \\
k
\end{array}\right] q^{k^{2}+\frac{1}{2} k}(-x)^{k}
$$

Here $\left[\begin{array}{c}N \\ k\end{array}\right]$ denotes the $q$-binomial which is defined by

$$
\left[\begin{array}{c}
N \\
k
\end{array}\right]=\frac{(q)_{N}}{(q)_{k}(q)_{N-k}}, \quad(q)_{n}=\prod_{j=1}^{n}\left(1-q^{j}\right) .
$$

We can also consider the multi-point function of B-branes. From the general formula of correlator of determinants [22], the $K$-point function of B-branes reads

$$
\Delta(x)\left\langle\prod_{i=1}^{K} B\left(x_{i}\right)\right\rangle=\operatorname{det}\left(\mathcal{S}_{N+j-1}\left(x_{i}\right)\right) .
$$

\subsection{A Map from A-brane Amplitudes to B-brane Amplitudes}

Now we consider the relation between the A-brane amplitude $\mathcal{Z}_{N}(x)=\left\langle\Psi_{D}(x)\right\rangle$ in the crystal picture and the mirror B-brane amplitude $\mathcal{S}_{N}(x)=\langle B(x)\rangle$ in the Chern-Simons matrix model. To see this relation, let us rewrite $\mathcal{Z}_{N}(x)$ given in the product form (3.3) into a summation form [19]

$$
\mathcal{Z}_{N}(x)=\sum_{k=0}^{N}\left[\begin{array}{c}
N \\
k
\end{array}\right] q^{\frac{1}{2} k(k+1)}(-x)^{k}
$$

By comparing this expression with $\mathcal{S}_{N}(x)$ in (3.12), one can immediately see that they are almost identical except for the exponent of $q$. This difference is taken care of by the following integral

$$
\int_{-\infty}^{\infty} \frac{d s}{\sqrt{2 \pi g_{s}}} e^{-\frac{1}{2 g_{s}} s^{2}} e^{i k s}=q^{\frac{1}{2} k^{2}}
$$

Therefore, $\mathcal{S}_{N}(x)$ and $\mathcal{Z}_{N}(x)$ are related by

$$
\mathcal{S}_{N}(x)=\int_{-\infty}^{\infty} \frac{d s}{\sqrt{2 \pi g_{s}}} e^{-\frac{1}{2 g_{s}} s^{2}} \mathcal{Z}_{N}\left(x e^{i s}\right)
$$

4 In [11], a unitary matrix model with measure $\vartheta_{3}\left(q, e^{i \varphi}\right)$ is considered. The orthogonal polynomials on $S^{1}$ associated with this weight are known as the Rogers-Szegö polynomials [19]. They are related to the Stieltjes-Wigert polynomials by a change of variable.

5 This is a special case of the $q$-binomial theorem [27]. 
As advertised, this is nothing but the integral transformation $\mathcal{Q}$ appeared in the Gaussian matrix model! (Recall that the variable obeying the Gaussian distribution is $\log x$ in the Chern-Simons matrix model. Therefore, the transformation (3.17) is exactly the same as $\mathcal{Q}$ in (2.6).) To summarize, the A-brane amplitude and the B-brane amplitude are related by the map $\mathcal{Q}$

$$
\mathcal{Q}: \mathcal{Z}_{n}(x) \mapsto \mathcal{S}_{n}(x)
$$

We can easily generalize this relation to the multi-point correlators. Let us consider the 2-point function for illustration. The 2-point function of A-branes is (see (3.6))

$$
\left\langle\prod_{i=1}^{2} \Psi_{D}\left(x_{i}\right)\right\rangle=\left(x_{2}-x_{1}\right) \mathcal{Z}_{N}\left(x_{1}\right) \mathcal{Z}_{N}\left(x_{2}\right)
$$

Using the recursion relation of $\mathcal{Z}_{N}(x)$

$$
x q^{N+1} \mathcal{Z}_{N}(x)=\mathcal{Z}_{N}(x)-\mathcal{Z}_{N+1}(x)
$$

(3.19) is rewritten as

$$
\left\langle\prod_{i=1}^{2} \Psi_{D}\left(x_{i}\right)\right\rangle=q^{-N-1}\left[\mathcal{Z}_{N+1}\left(x_{1}\right) \mathcal{Z}_{N}\left(x_{2}\right)-\mathcal{Z}_{N}\left(x_{1}\right) \mathcal{Z}_{N+1}\left(x_{2}\right)\right]
$$

By applying the $\mathcal{Q}$-map (3.18), this becomes the 2-point function of B-branes (3.14), up to an overall factor $q^{-N-1}$. One can easily generalize this argument for the general $K$-point function, and it is found to be

$$
\left\langle\prod_{i=1}^{K} \Psi_{D}\left(x_{i}\right)\right\rangle=\operatorname{det}\left(\mathcal{Z}_{N+j-1}\left(x_{i}\right)\right)
$$

Here we suppressed an overall $q$ dependent factor for simplicity. Clearly, (3.22) is mapped to (3.14) by the $\mathcal{Q}$-map (3.18). In this way we find that the A-brane correlator and the B-brane correlator are related by the $\mathcal{Q}$-map

$$
\Delta(x)\left\langle\prod_{i=1}^{K} B\left(x_{i}\right)\right\rangle=\int_{-\infty}^{\infty} \prod_{i=1}^{K} \frac{d s_{i}}{\sqrt{2 \pi g_{s}}} e^{-\frac{1}{2 g_{s}} s_{i}^{2}}\left\langle\prod_{j=1}^{K} \Psi_{D}\left(x_{j} e^{i s_{j}}\right)\right\rangle .
$$

Recalling the form of A-brane correlator (3.6), this relation is written in a similar form as the Gaussian matrix model case 2.13

$$
\mathcal{Q}: \Delta(x) \prod_{i=1}^{K} \mathcal{Z}_{N}\left(x_{i}\right) \mapsto \Delta(x)\left\langle\prod_{i=1}^{K} B\left(x_{i}\right)\right\rangle .
$$


Finally, if we introduce the $\mathcal{Q}$-transform $\mathcal{Q} \Psi_{D}$ of A-brane operator $\Psi_{D}$, (3.23) can be written in a more suggestive form

$$
\Delta(x)\left\langle\prod_{i=1}^{K} B\left(x_{i}\right)\right\rangle=\left\langle\prod_{i=1}^{K} \mathcal{Q} \Psi_{D}\left(x_{i}\right)\right\rangle .
$$

Note that $\mathcal{Q} \Psi_{D}(x)$ is smeared by the Gaussian integral with width $g_{s}$, thus it is no longer a local operator in the $x$-space. Perhaps it might be related to the noncommutativity in spacetime.

\section{Discussion}

In this paper, we found that the A-brane correlators and the B-brane correlators are related by the transformation $\mathcal{Q}$, which is just a Gaussian integral. We would like to understand the physical meaning of this relation. By the analogy to the Gaussian matrix model case, it seems natural to regard the A-brane amplitude $\mathcal{Z}_{N}(x)$ as a classical value of the determinant in the Chern-Simons matrix model. From the product form of $\mathcal{Z}_{N}(x)$ (3.3), the classical background $M_{0}$ corresponding to $\mathcal{Z}_{N}(x)$ is given by

$$
\begin{gathered}
\mathcal{Z}_{N}(x)=(-1)^{N} q^{\frac{1}{2} N(N+1)} \operatorname{det}\left(x-M_{0}\right), \\
M_{0}=\operatorname{diag}\left(q^{-1}, q^{-2}, \cdots, q^{-N}\right) .
\end{gathered}
$$

In other words, $\mathcal{Z}_{N}(x)$ vanishes at

$$
\log x=g_{s}, 2 g_{s}, \cdots, N g_{s}
$$

i.e., the zeros of $\mathcal{Z}_{N}(x)$ are equally spaced.6 This is consistent with the picture that the A-brane probes the background lattice of the crystal. Namely, the A-brane amplitude obtained in the crystal picture is a probe approximation ignoring the backreaction. Moreover, it is known [18,12] that the A-brane amplitude is the zero energy wavefunction of the Hamiltonian $H\left(u,-g_{s} \partial_{u}\right)$, where $H(u, v)$ defines the mirror Riemann surface $H(u, v)=0$. In our case, $\mathcal{Z}_{N}\left(e^{u}\right)$ is the zero-mode of the Hamiltonian

$$
H(u, v)=1-e^{u}-\left(1-e^{u} q^{N}\right) e^{v} .
$$

6 In [28], the eigenvalue density of Chern-Simons matrix model is studied numerically at finite $N$. It is observed that the density has $N$ peaks with almost equal spacing. This is consistent with our identification of $M_{0}$ as the classical configuration of Chern-Simons matrix model. 
This agrees with the known mirror of the resolved conifold [15,29] up to a linear canonical transformation of the $u, v$ coordinates [18].

On the other hand, the B-brane amplitude $\mathcal{S}_{N}(x)$ includes the effect of deformation of closed string background due to the presence of D-brane [18]. In fact, the insertion of determinants is equivalent to a certain shift of matrix model potential [18,20]

$$
\left\langle\prod_{i} \operatorname{det}\left(x_{i}-M\right)\right\rangle=\frac{1}{Z_{C S}} \int d M \exp \left[-\frac{1}{2 g_{q}} \operatorname{Tr}(\log M)^{2}+\sum_{i} \operatorname{Tr} \log \left(x_{i}-M\right)\right]
$$

As argued in [18], the deformation of matrix model potential due to the insertion of Bbranes corresponds to turning on gravitational descendants of Kähler class of $\mathbb{P}^{1}$. It is remarkable that this deformation is captured by the simple map $\mathcal{Q}$. Another way to see this effect is to look at the zeros of $\mathcal{S}_{N}(x)$. For example, $\mathcal{S}_{2}(x)$ is factorized as

$$
\begin{aligned}
\mathcal{S}_{2}(x) & =1-q^{\frac{3}{2}}(1+q) x+q^{5} x^{2}=q^{5}\left(x-\alpha_{+}\right)\left(x-\alpha_{-}\right) \\
\alpha_{ \pm} & =\frac{1}{2} q^{-\frac{7}{2}}[1+q \pm \sqrt{(1-q)(1+3 q)}]
\end{aligned}
$$

The zeros are no longer equally spaced. This might be seen as the distortion of crystal due to the insertion of brane. One might expect that the "quantum" wavefunction $\mathcal{S}_{N}(x)$ is significantly different from its classical counterpart $\mathcal{Z}_{N}(x)$. It would be interesting to study the large $N$ behavior of $\mathcal{S}_{N}(x)$. It would be also interesting to consider a double-scaling limit of Chern-Simons matrix model as in the case of Gaussian matrix model [20].

In this paper we did not consider the insertion of anti-brane. In the crystal picture, it is known how to construct the anti-brane operator $\Psi_{\bar{D}}[10$. However it is not clear to us what is the corresponding operator in the Chern-Simons matrix model. We leave this as a future problem.

Acknowledgment: I would like to thank Marcos Marino for correspondence. 


\section{References}

[1] R. Gopakumar and C. Vafa, "Topological gravity as large N topological gauge theory," Adv. Theor. Math. Phys. 2, 413 (1998) arXiv:hep-th/9802016.

[2] R. Gopakumar and C. Vafa, "On the gauge theory/geometry correspondence," Adv. Theor. Math. Phys. 3, 1415 (1999) arXiv:hep-th/9811131.

[3] E. Witten, "Quantum Field Theory And The Jones Polynomial," Commun. Math. Phys. 121, 351 (1989).

[4] M. Bershadsky, S. Cecotti, H. Ooguri and C. Vafa, "Kodaira-Spencer theory of gravity and exact results for quantum string amplitudes," Commun. Math. Phys. 165, 311 (1994) arXiv:hep-th/9309140.

[5] E. Witten, "Chern-Simons gauge theory as a string theory," Prog. Math. 133, 637 (1995) arXiv:hep-th/9207094.

[6] H. Ooguri and C. Vafa, "Worldsheet derivation of a large N duality," Nucl. Phys. B 641, 3 (2002) arXiv:hep-th/0205297.

[7] H. Ooguri and C. Vafa, "Knot invariants and topological strings," Nucl. Phys. B 577, 419 (2000) arXiv:hep-th/9912123.

[8] A. Okounkov, N. Reshetikhin and C. Vafa, "Quantum Calabi-Yau and classical crystals," arXiv:hep-th/0309208.

[9] A. Iqbal, N. Nekrasov, A. Okounkov and C. Vafa, "Quantum foam and topological strings," arXiv:hep-th/0312022.

[10] N. Saulina and C. Vafa, "D-branes as defects in the Calabi-Yau crystal," arXiv:hepth/0404246.

[11] T. Okuda, "Derivation of Calabi-Yau crystals from Chern-Simons gauge theory," JHEP 0503, 047 (2005) arXiv:hep-th/0409270].

[12] R. Dijkgraaf, A. Sinkovics and M. Temurhan, "Universal correlators from geometry," JHEP 0411, 012 (2004) [arXiv:hep-th/0406247.

[13] N. Halmagyi, A. Sinkovics and P. Sulkowski, "Knot invariants and Calabi-Yau crystals," JHEP 0601, 040 (2006) arXiv:hep-th/0506230.

[14] M. Marino, "Chern-Simons theory, matrix integrals, and perturbative three-manifold invariants," Commun. Math. Phys. 253, 25 (2004) arXiv:hep-th/0207096.

[15] M. Aganagic, A. Klemm, M. Marino and C. Vafa, "Matrix model as a mirror of Chern-Simons theory," JHEP 0402, 010 (2004) arXiv:hep-th/0211098.

[16] M. Tierz, "Soft matrix models and Chern-Simons partition functions," Mod. Phys. Lett. A 19, 1365 (2004) arXiv:hep-th/0212128.

[17] M. Marino, "Les Houches lectures on matrix models and topological strings," arXiv:hep-th/0410165.

[18] M. Aganagic, R. Dijkgraaf, A. Klemm, M. Marino and C. Vafa, "Topological strings and integrable hierarchies," Commun. Math. Phys. 261, 451 (2006) arXiv:hepth/0312085]. 
[19] G. Szegö, Orthogonal Polynomials, Colloquium Publications, Vol. 23 (American Mathematical Society).

[20] J. M. Maldacena, G. W. Moore, N. Seiberg and D. Shih, "Exact vs. semiclassical target space of the minimal string," JHEP 0410, 020 (2004) arXiv:hep-th/0408039.

[21] D. Gaiotto and L. Rastelli, "A paradigm of open/closed duality: Liouville D-branes and the Kontsevich model," JHEP 0507, 053 (2005) arXiv:hep-th/0312196].

[22] A. Morozov, "Integrability and matrix models," Phys. Usp. 37, 1 (1994) arXiv:hepth/9303139|.

[23] M. Aganagic and C. Vafa, "Mirror symmetry, D-branes and counting holomorphic discs," arXiv:hep-th/0012041.

[24] M. Aganagic, A. Klemm and C. Vafa, "Disk instantons, mirror symmetry and the duality web," Z. Naturforsch. A 57, 1 (2002) arXiv:hep-th/0105045.

[25] L. D. Faddeev and R. M. Kashaev, "Quantum Dilogarithm," Mod. Phys. Lett. A 9, 427 (1994) arXiv:hep-th/9310070.

[26] P. J. Forrester, "Vicious random walkers in the limit of a large number of walkers," J. Stat. Phys. 56, 767 (1989).

[27] C. E. Andrews, The Theory of Partitions (Cambridge University Press, 1998).

[28] S. de Haro and M. Tierz, "Discrete and oscillatory matrix models in Chern-Simons theory," Nucl. Phys. B 731, 225 (2005) arXiv:hep-th/0501123].

[29] K. Hori and C. Vafa, "Mirror symmetry," arXiv:hep-th/0002222. 